\title{
Left-handed Transmission Line Mass Flow Meter for Particulate Solids in Pipelines
}

\author{
Andreas Penirschke, Margarita Puentes, Martin Schüßler and Rolf Jakoby \\ Technische Universität Darmstadt, Microwave Engineering,Merckstr. 25, D-64283 Darmstadt, Germany, \\ Phone: +49615116 3162, Fax: +49615116 4322, \\ Email: penirschke@mwt.tu-darmstadt.de.
}

\begin{abstract}
A particulates mass flow meter has been developed using a composite right/left-handed transmission line resonator. The presented sensor principle promises the possibility to design precise particulate solids mass flow meter with moderate costs. The spatial filtering velocimetry function is applied to the CRLHresonator in order to detect the velocity information and the material density from a single measurement. The application areas of these sensors are gas/solids, gas/liquid and liquid/solids flows in various industrial monitoring applications.
\end{abstract}

\section{INTRODUCTION}

The accurate mass flow measurement of particulate solids or liquids in pipelines using microwave methods is a recent field of research [1]. Besides mechanical-, acoustic- and electrostatic sensors, also microwave techniques are used for this field of application [2]-[7]. Radar systems, where a pulse or a Continuous Wave-radar signal is radiated perpendicular to the material flow into the measurement section are promising microwave techniques. Drawbacks of these techniques are moderate detection accuracy and relatively high costs of such radar systems. Other microwave systems use TM- and TE- modes inside the metallic pipeline to detect the dielectric load within the tube [3]-[6]. These techniques offer high detection accuracies for the material density but, up to now, no velocity information. The operating frequency of these systems has to be in the order of the pipelines-cutoff frequency or higher. A new

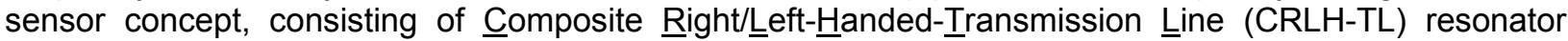
reduces the necessary operating frequency and sensor length. In this concept series capacitors and shunt inductors are used with simultaneously negative permeability and permittivity - the so called lefthanded metamaterials, that have been demonstrated superior properties [8], [9] for several applications. This paper investigates an application of this concept for a compact mass flow sensor with increased sensitivity compared to a conventional microwave mass flow sensor. A prototype of a particulate mass flow sensor for a pneumatic conveying system [10] was built up and measurements were done to proof the concept.

\section{SENSOR OVERVIEW}

The sensor setup consists of the signal detection (Part A), that detects the particulate flow in the pipeline, the material concentration detection (Part B) and the velocity detection (Part C). Fig. 1 displays the block diagram of the complete sensor system. The signal detection (Part A) is presented in section III. Here, the RF-signal, generated by the RF-power source is splitted by a power divider into two signals, one connected to the CRLH-TL-resonator and one connected to a phase shifter. The RF-signal obtained from the CRLH-TL-resonator is connected to one port of the phase comparator and the output of the phase shifter to the other one. If no particulate solids are fed, the setup can be calibrated to a zero phase shift $\Delta \varphi$ between both comparator signals. For a particulate flow in the pipeline, it will change and the phase comparator exhibits the phase difference versus time $\varphi(t)$. For a particulate flow in the pipeline a non zero phase shift occurs. The phase comparator gives the phase difference versus time $\varphi(t)$. The mean value of $\varphi(t)$ contains the material concentration information. Section IV explains the material density extraction algorithm (Part B). After high pass filtering (Part C), $\varphi(t)$ contains the velocity information of the material flow. The velocity extraction algorithm is presented in section $\mathrm{V}$. In order to extract the velocity information the spatial filtering velocimetry method is applied to the standing waves in the measurement section. 


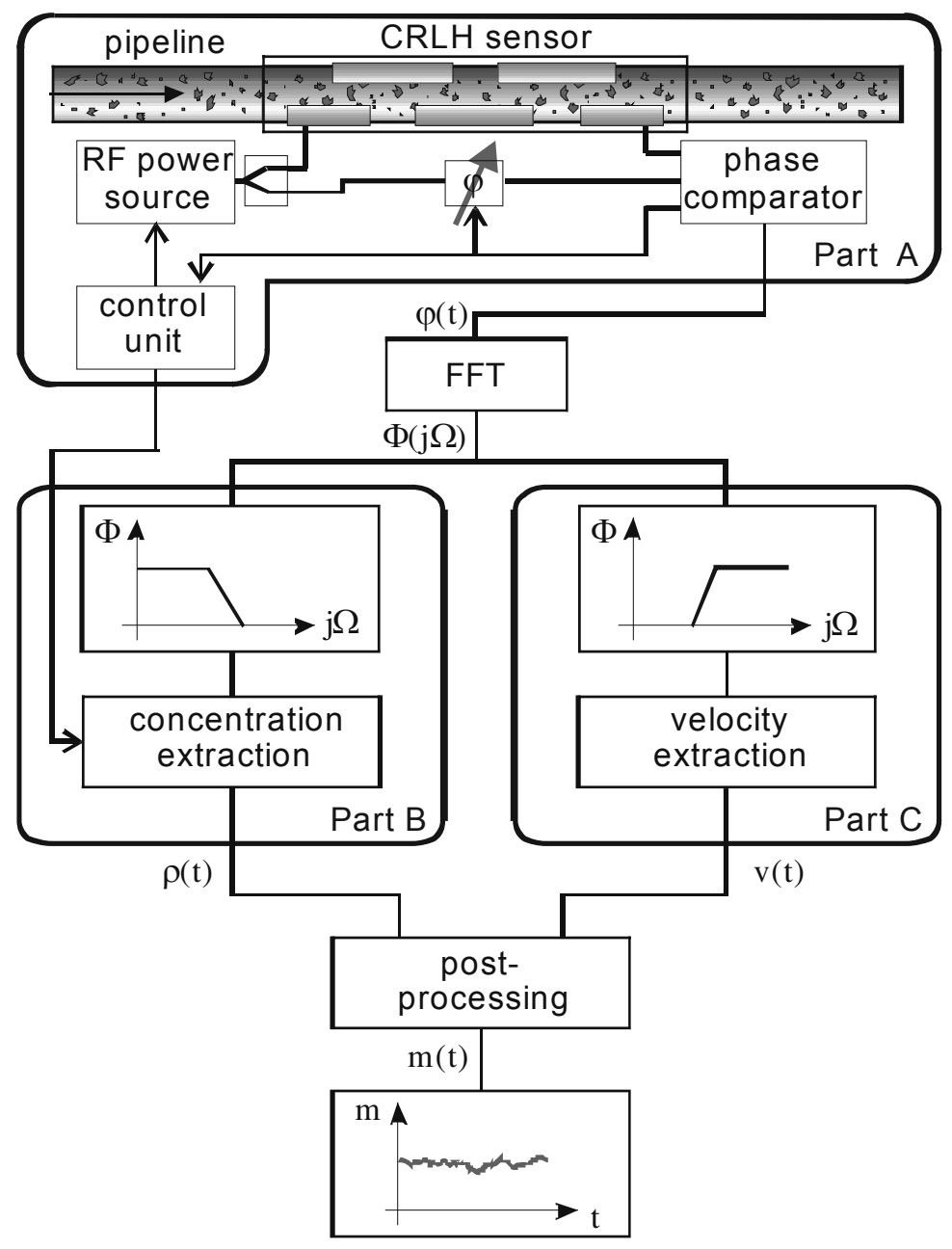

Fig. 1. Block-diagram of the complete sensor setup.

\section{COMPOSITE RIGHT/LEFT-HANDED TRANSMISSION LINE RESONATORS}

\section{A. CRLH-TL-SENSOR DESIGN}

A well known way to detect the permittivity of a solid material is to use a resonant circuit consisting of a capacitor and an inductor. A change in the permittivity of the capacitor causes a change in its capacitance and hence a shift of the circuit resonant frequency. The proposed mass flow sensor principle uses resonant circuits based on CRLH-transmission lines.

A lossless CRLH-TL can be described by its equivalent circuit (shown in Fig. 2). $L_{R H / L H}$ and $C_{R H / L H}$ represent the inductance and capacitance times unit lenght and per unit length $\Delta z$, respectively.

The propagation constant for the CRLH-TL is given by

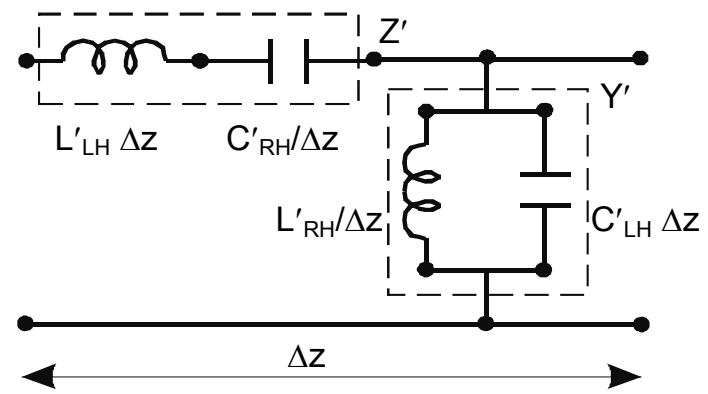

Fig. 2. Equivalent circuit model for an infinitesimal short lossless CRLH-TL.

$$
\gamma=\alpha+j \beta=\sqrt{Z^{\prime} Y^{\prime}},
$$

where $Z^{\prime}$ and $Y^{\prime}$ are the impedance and admittance of the TL and they are defined as 


$$
\begin{aligned}
& Y^{\prime}(j \omega)=j\left(\omega \mathrm{L}_{\mathrm{RH}}^{\prime}-\frac{1}{\omega \mathrm{C}_{\mathrm{LH}}^{\prime}}\right), \\
& Z^{\prime}(j \omega)=j\left(\omega \mathrm{C}_{\mathrm{RH}}^{\prime}-\frac{1}{\omega \mathrm{L}_{\mathrm{LH}}^{\prime}}\right) .
\end{aligned}
$$

The phase constant, or dispersion relation $\beta(j \omega)$ is

$$
\beta(j \omega)= \pm \sqrt{\omega^{2} L_{R H}^{\prime} C_{R H}^{\prime}+\frac{1}{\omega^{2 L_{L H}^{\prime} C_{L H}^{\prime}}}-\left(\frac{L_{R H}^{\prime}}{L_{L H}^{\prime}}+\frac{C_{R H}^{\prime}}{C_{L H}^{\prime}}\right)},
$$

where $\beta(j \omega)$ is a real value for the RH-passband $(\beta>0)$ and the LH-passband $(\beta<0)$. $\beta$ becomes imaginary outside the two pass-bands and therefore stop-bands occur. An unbalanced CRLH-TL resonator design where higher order modes are considered and $Z_{0, L H}$ is much larger than $Z_{0, R H}$, allows the design of sensitive permittivity sensors if the absolute changes of the detected permittivity are small [6].

For a constant measurement frequency the phase shift as a function of the permittivity change can be approximated by

$$
\Delta \varphi=\frac{\pi}{2} \cdot \mathrm{Q} \cdot \frac{\Delta \varepsilon_{\mathrm{r}}}{2} \text { for } \Delta \varepsilon_{\mathrm{r}} / \varepsilon_{\mathrm{r}, \mathrm{ref}} \ll 1,
$$

where $Q$ is the loaded $Q$-factor of the resonant circuit and $\Delta \varepsilon r$ is the difference $\varepsilon_{r, \text { meas }}-\varepsilon_{r, \text { ref }}$. A system sensitivity $s$ can be defined as

\section{B. CRLH-SENSOR PROTOTYPE}

$$
\mathrm{s}=\frac{\Delta \varphi}{\Delta \varepsilon_{\mathrm{r}}}=\mathrm{Q} \cdot \frac{\pi}{4}
$$

The cross section of the cylindrical measurement setup of the LC-loaded CRLH-TL (based on a LCloaded microstrip CRLH-TL principle [10]) is shown in Fig. 3a.

The material density CRLH-sensor consists of a dielectric conveying tube with an inner diameter di, Metal Insulate Metal (MIM) capacitances $\mathrm{C}_{\mathrm{LH}}$ assembled around the tube and wire $L_{L H}$ from the capacitor plates to ground. The $\mathrm{C}_{\mathrm{RH}}$ takes place from the $\mathrm{C}_{\mathrm{LH}}$ plates to the ground layer and the LRH from the $\mathrm{C}_{\mathrm{LH}}$ plates. The ground layer is a metallic tube with the diameter do. The schematic cross section of one MIM $\mathrm{C}_{\mathrm{LH}}$ is shown in Fig. 3b.

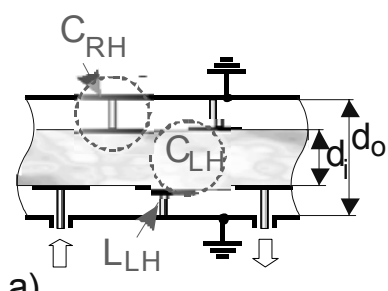

a)

Fig. 3. CRLH-sensor: Schematic cross section of a microstrip line resonator assembled around a dielectric conveying tube (a) and cut out of a MIM capacitor (b).

The lumped element values were determined by a deembeding procedure using a circuit simulator and prove the concept of the CRLH-TL-sensor as shown in Fig $4 a$.
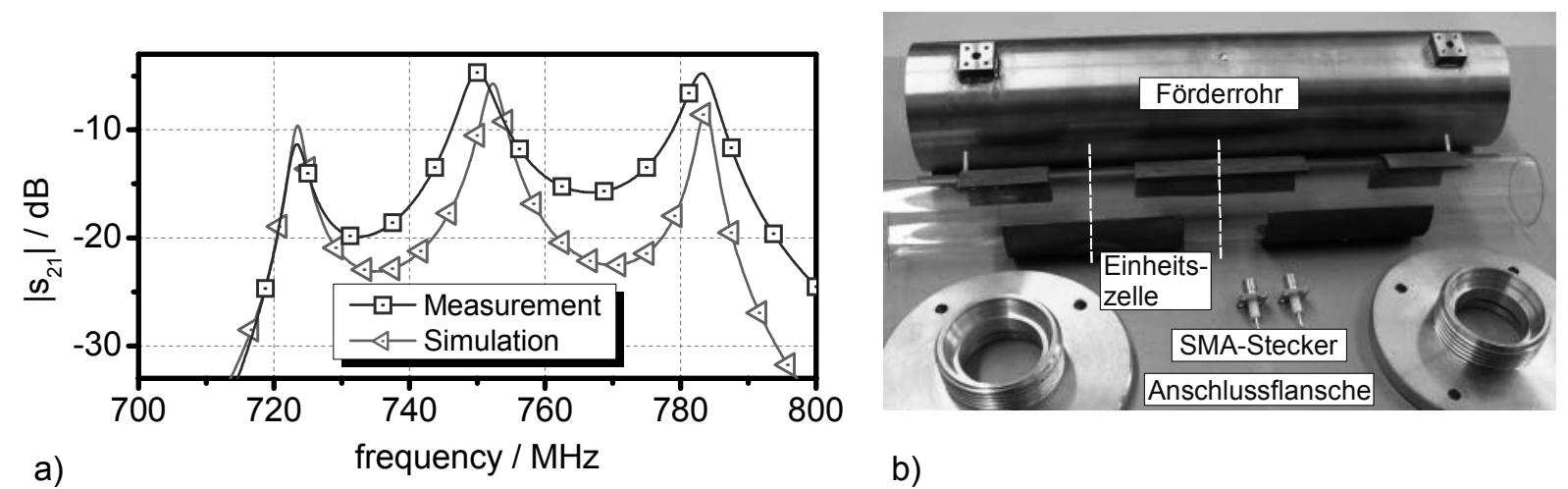

b)

Fig. 4. a) Measurement results compared with circuit simulator results and b) Picture of the disassembled CRLH-sensor.

Fig. $4 \mathrm{~b}$ shows a picture of the disassembled CRLH-sensor. The pipeline section consists of low-loss duran glass with an inner diameter of $32 \mathrm{~mm}$. MIM LH-capacitors assembled around the tube and wire $\mathrm{LH}$-inductors from the capacitor plates to ground. Stray $\mathrm{RH}$-capacitances exist from the LH-capacitor 
plates to the ground layer and stray $\mathrm{RH}$-inductances along the $\mathrm{LH}$-capacitor plates. The ground layer is an outer conducting tube with a diameter of $60 \mathrm{~mm}$.

\section{MATERIAL CONCENTRATION EXTRACTION}

A change in the permittivity of the material, or the material density of the particulate solids feeded through the measurement section causes a change in the $\mathrm{C}_{\mathrm{LH}}$ capacitances and hence a shift of the circuits resonant frequency. Close to the resonant frequency the phase gradient reaches its maximum and remains constant so that the resonance shift is proportional to the sensors phase shift. The material density is a function of the mean value of the detected phase shift $\varphi_{\text {meas }}(t)$. The mean value can either be taken from the integration of $\varphi_{\text {meas }}(t)$ or from the zero value of the Fourier Transform of $\varphi_{\text {meas }}(t)$. Together with the unloaded phase shift $\varphi_{0}(\mathrm{t})$ and the loaded $\mathrm{Q}$-factor of the empty measurement section, the effective absolute permittivity can be approximated by

$$
\varepsilon_{\mathrm{r}, \text { eff }}^{\prime}(\mathrm{t}) \approx\left(1-\frac{\varphi_{0}-\varphi_{\text {meas }}(\mathrm{t})}{\mathrm{Q}_{\mathrm{L}}}\right)^{2}
$$

The concentration $\alpha$ of the solids (in the solid/air mixture) in the measurement section can be defined as:

$$
\alpha=\frac{\mathrm{V}_{\text {MUT }}}{\mathrm{V}_{\text {Air }}+\mathrm{V}_{\text {MUT }}}
$$

With the assumption that $\varepsilon_{r, \text { eff }}$ is a linear function of the solids concentration, follows

$$
\varepsilon_{\mathrm{r}, \mathrm{eff}}^{\prime}=\varepsilon_{\mathrm{r}, \mathrm{MUT}}^{\prime} \cdot \alpha+\varepsilon_{\mathrm{r}, \mathrm{Air}}^{\prime} \cdot(1-\alpha)
$$

If the specific density of the bulk material MUT is known, the integral value of the material concentration can be calculated by

$$
\rho_{\text {eff }}(t) \approx \rho_{\text {MUT }} \cdot \frac{\varepsilon_{\text {r,eff }}^{\prime}(t)-\varepsilon_{r, A i r}^{\prime}}{\varepsilon_{r, \text { MUT }}^{\prime}-\varepsilon_{r, \text { eff }}^{\prime}(t)},
$$

where $\varepsilon_{r, M U T}$ is the permittivity of the bulk material and $\varepsilon_{r, A i r}$ is the permittivity of the conveyed air (usually 1).

\section{VELOCITY DETECTION}

Most mass flow sensors for particulate solids, use mechanical velocity sensors, correlation- or Dopplertechniques. For conveyed powders, correlation based sensors as well as mechanical sensors are often not suitable to extract the velocity information precisely. To detect the velocity of such small particle sizes, the laser Doppler velocimetry method can be used. This technique has an extensive setup and needs two coherent lasers for velocity detection. The spatialfiltering velocimetry [9] adopts the principle of the laser Doppler velocimetry technique. The measurement setup consists of one light source and a spatial filter only.

Fig. 5a displays two examples for transmittance functions of illuminated spatial filters with grating lines for velocity measurements in the x-direction. The light intensity I, received by a photo detector, varies periodically due to object shadowing. The obtained signal depends on the velocity of the moving object and the properties of the spatial filter. For a rectangular transmittance the output signal is a rectangular function as shown on Fig. 5a. A sinusoidal transmittance results in a periodic output signal with a sinusoidal behavior (Fig. 5b).

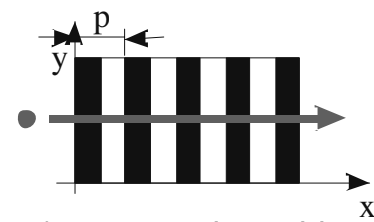

a) rectangular grid

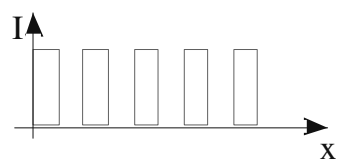

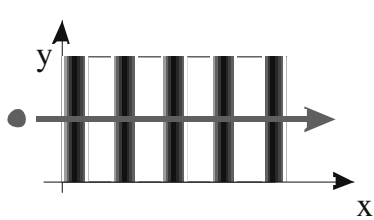

b) sinusodial grid

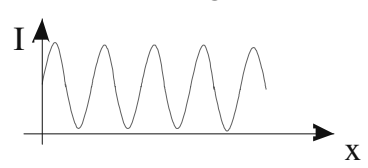

Fig. 5. Spatial-filtering velocimetry: Rectangular type transmission grating with a) rectangular- and b) sinusoidal transmittance.

\section{E. Implementation of spatial-filtering velocimetry in the microstrip patch detector}

In order to extract the velocity of the objects or pulverized solids flowing through the microstrip patch sensor, the change of the complex transmission $S_{21}(t)$ at a resonant mode was investigated and the spatial filtering velocimetry was applied [10].

If a single particle passes the measurement section, the time dependent phase shift describes three sinusoidal half waves. The time difference between two adjacent phase minima corresponds to the time $t$, which the particle needs to travel from one field maximum to the adjacent field minimum or vice versa. 
The particle position in the sensors cross section defines the maximum phase deviation at the electrical field maxima. If the particle passes the sensor in the middle of the cross section, the phase shift is larger than for the case when the particle passes the section at the outermost position of the pipelines crosssection.

\section{MEASUREMENT SETUP}

The realized test facility to analyze the mass flow sensor is shown in Fig. 6.

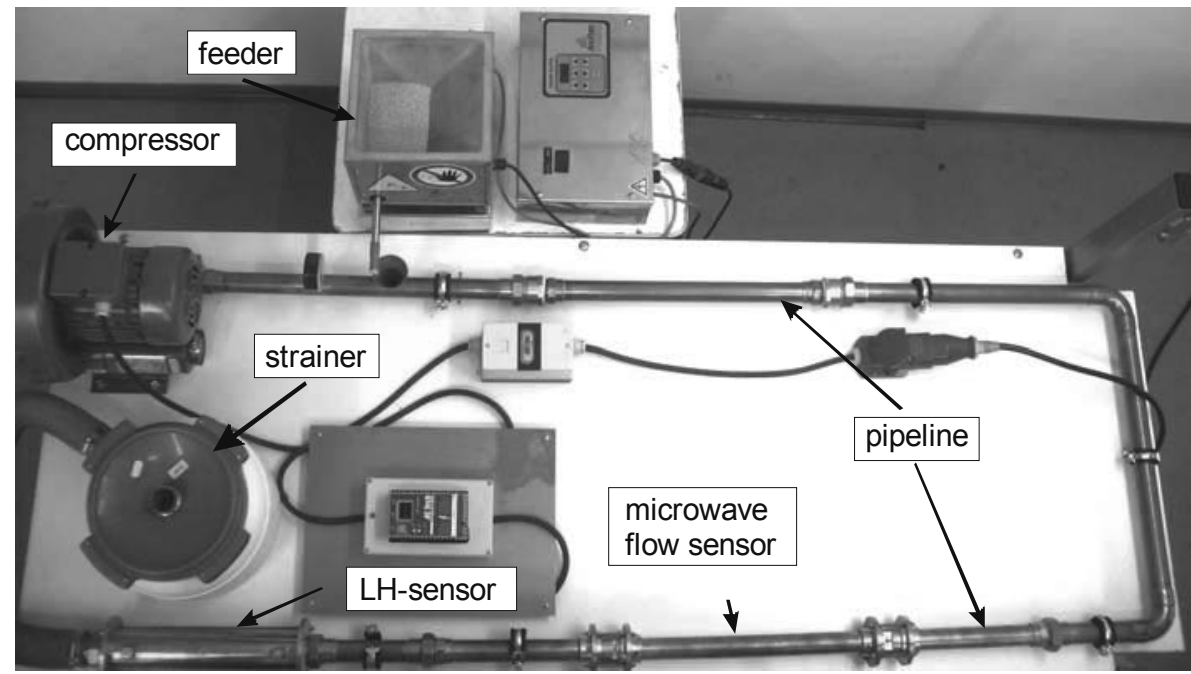

Fig. 6. Material flow test facility: Feed rates up to $1000 \mathrm{~g} / \mathrm{h}$ and velocities

It consists of a compressor, a venturi injector, a pipeline section and a strainer. The compressed air having a velocity of up to $25 \mathrm{~m} / \mathrm{s}$ reaches the venturi injector, where the material is fed into the pipeline by a loss in weight feeder. The material-air mixture is nearly homogeneously distributed when it reaches the sensor. After traveling through the sensor the mixture reaches the strainer, where the material is separated from the air.

\section{MEASUREMENT RESULTS}

As a proof-of-concept, measurements were carried out with the material flow test. Fig. 7 shows the results obtained from feeding material with a feed rate of $1100 \mathrm{~g} / \mathrm{h}$.

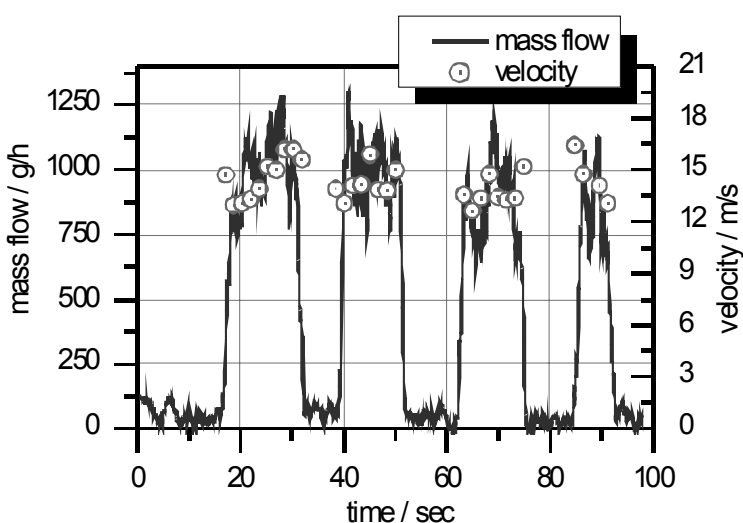

a) CRLH-Sensor

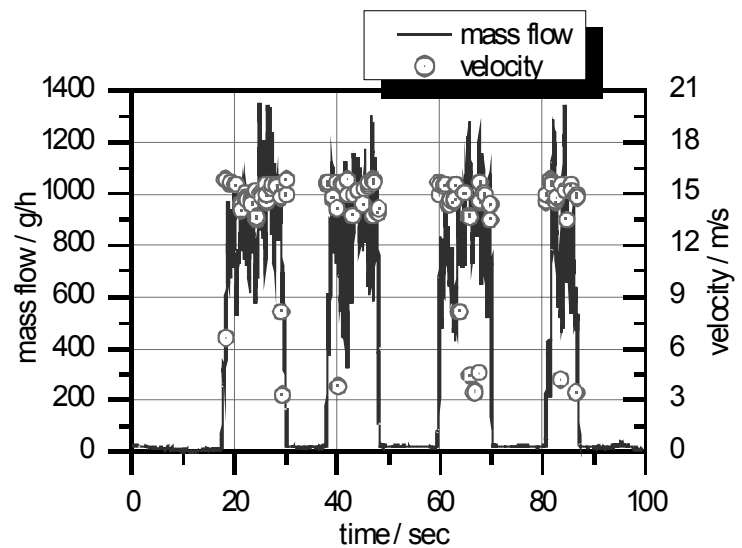

b) MSP-Sensor

Fig 7. a) Measurement results MSP-Sensor and b) Measurement results CRLH-Sensor.

The material density detected by the CRLH-sensor is shown in Fig. 7a. As expected, the detected particulate velocity is in the range of $15 \mathrm{~m} / \mathrm{s}$. The extracted mass flow rate diverges less than $10 \%$ from the original value of the loss in the weight feeder. Reference measurements were carried out with a 
Microstrip Patch sensor (MSP) [10]. Up to now only measurements with a constant feedrate of $1100 \mathrm{~g} / \mathrm{h}$ were carried out with a particulate velocity of $15 \mathrm{~m} / \mathrm{s}$. Two vector network analyzers were used to detect the signals for the MSP-sensor and the CRLH-sensor. Since the network analyzers were only able to detect samples in CW-mode for about 100s no long term measurements could be made up to now. The material density detected by the MSP-sensor is shown in Fig. 7b, where it can be seen that the feedrate was not constant and changes in the concentration of more than $10 \%$ were obtained. The velocity detected by the MSP-sensor differ less than $\pm 5 \%$ from the nominal value. The results obtained for the CRLH-sensor are shown in Fig. 10. Since the averaging of the concentration detection was higher, a more smoother curve was obtained. For the $\mathrm{CRLH}$-sensor a smoother curve was obtain due to the averaging in the concentration detection. The results differ less that $5 \%$ from the concentration obtained with the MSP-sensor. The detected velocity differ more that $10 \%$ from the original value.

\section{CONCLUSION \& OUTLOOK}

A new mass flow density sensor for process monitoring applications based on a Composite Right/LeftHanded transmission line resonator was presented. Compared to microwave sensors [3], [7] that use frequencies in the order of the pipelines cutoff frequency the CRLH-TL sensor principle can be designed for lower frequencies. There is always a trade-off between the maximum possible sensor length, the operation frequency, the sensitivity and the costs of such a system. Increasing the sensor length causes mechanical instabilities as well as a reduction of the spatial resolution, whereas increasing the frequency results in higher costs of the sensor system. The CRLH-TL resonator meets the requirements of a short sensor length, low frequencies to reduce the sensor costs and an adequate sensitivity to detect small changes in the permittivity. The spatial filtering velocimetry offers the possibility to detect the material velocity from a single sensor if its field distribution is known. The accuracy of the velocity measurements is determined substantially by the ability of the signal processing system employed.

\section{REFERENCES}

[1] Y. Yan, and D. Stewart, "Guide to the Flow measurement of Particulate Solids in Pipelines", published by the Institute of Measurement and Control, National Engineering Laboratory, and the University of Greenwich, April 2001.

[2] A. Penirschke, M. Schüßler, and R. Jakoby, "New Microwave Flow Sensor based on a Left-Handed Transmission Line Resonators", 2007 MTT Int. Microwave Symp. Dig, June 2007.

[3] H. Maune, M. Schüßler, A. Penirschke, C. Damm, and R. Jakoby "Left-Handed Transmission Line for Permittivity Measurements", Metamaterials Conference 2007.

[4] A. Penirschke, J. Freese, J. Biebel, B. Allenberg, and R. Jakoby, "Novel integrated coaxial line to cylindrical waveguide directive couplers in pipelines for process monitoring applications", 2005 MTT Int. Microwave Symp. Dig., June 2005, pp. 1203-1206.

[5] A. Penirschke, B. Allenberg, and R. Jakoby, "Antenna configuration for the transition in and out of tubular hollow structures and method for the measurement of the mass flow rate using such configurations", German Patent 102004057087 B3, Jan. 19, 2006.

[6] A. Penirschke, and R. Jakoby, "Accurate Material Density Measurements of Particulate Solids in Pipelines with a Novel Microwave Flow Sensor", 13. ITG/GMA Fachtagung Sensoren und Messsysteme 2006, Freiburg/Breisgau, March 2006.

[7] H. G. Conrads, "Method and device for a contact-free measurement of the mass flow rate in a two phase pneumatic transport using microwaves," EP. Patent 0717269 A2, Nov. 24, 1995.

[8] M. Schüßler, J. Freese, and R. Jakoby, "Design of Compact Planar Antennas using LHtransmission lines", 2004 MMT-S Int. Microwave Symp. Dig., vol. 1, pp. 209-212, June 2004.

[9] C. Caloz, and T. Itoh, "Novel Microwave Devices and Structures Based on the Transmission Line Approach of Meta-Materials", 2003 MTT-S Int. Microwave Symp. Dig., vol. 1, pp. 195-198, June 2003.

[10] A. Penirschke, and R. Jakoby, "Microwave Mass Flow Detector for Particulate Solids based on Spatial Filtering Velocimetry", 2008 IEEE Transactions on Microwave Theory and Techniques, vol. 56, pp. 3193 - 3199, Dec. 2008.

[11] Y. Aizu and T. Asakura, "Spatial Filtering Velocimetry", Springer Verlag, Berlin, 2006. 